\title{
Considerazioni su Antonio Gramsci
}

\author{
Elisa Marani \\ Middlebury College \\ maranielisa@gmail.com
}

\begin{abstract}
The aim of this essay is to recall the figure and thought of Antonio Gramsci through biographical aspects relevant to an assessment in his entirety (from his childhood spent in the land of Sardinia to the agitated years in Turin, from the communist militancy to the encounter with Tania, from the arrest to the years of imprisonment). Once imprisoned, Gramsci would elaborate an ambitious project working incessantly on it, while he would be gradually abandoned having become an inconvenience for most of his party comrades and his wife (this latter issue has yet to be clarified). A story of passion - civil, human, in a word political, but also a story of pain, suffering, and extreme loneliness the founder of the Italian Communist Party, whose pen became his perennial struggle for the "Common Good". I also want to draw attention to the persistent gap in the Italian cultural scene between the majority of the leftist intellectual class and the people. The leftist intellectuals often fall into a dangerous contradiction, and instead of filling the gap, they reinforce it often using not the best language to arrive to people. That is why despite years spent in studying Gramsci, and despite rhetorically recalling the great thinker, they fail in their mission as "gramscian intellectuals" who should have an ethical duty to approach the lower classes, instilling awareness and providing tools to get a real knowledge of the reality in order to change it.
\end{abstract}

Key words: Gramsci. Militancy. Imprisonment. Solitude. Gap.

Abstract: Con il presente articolo intendo anzitutto ricordare la figura e il pensiero di Antonio Gramsci ripercorrendone aspetti biografici importanti per 
valutare l'uomo nella sua interezza. Seguono gli episodi cruciali che hanno contribuito alla sua formazione intellettuale: dall'infanzia trascorsa nella terra sarda ai concitati anni nella Torino universitaria e proletaria, dall'incontro con Tania alla militanza comunista, dall'arresto agli anni del carcere. Proprio nel luogo di massima costrizione fisica Gramsci elabora l'ambizioso sogno comune lavorandovi incessantemente, mentre viene progressivamente abbandonato da molti dei compagni di partito e dalla moglie (vicenda, quest'ultima mai definitivamente chiarita). Una storia di forte passione- civile, umana, in una parola politica- da un lato, ma anche una storia di dolore, sofferenze e estrema solitudine quella del fondatore del Partito Comunista Italiano che nella penna trova il riscatto per una lotta perenne. L'obiettivo di questo intervento è anche quello di richiamare l'attenzione sul persistente divario, nel panorama culturale-politico italiano, tra certa intellettualità che si professa di sinistra e il popolo, e sulla palese contraddizione quindi di una classe-casta che non si impegna per ricomporre il gap. Questa mancata volontà della sinistra è esattamente il contrario di ciò che avrebbe voluto il grande pensatore cui pure retoricamente a volte si ritorna: l'intellettuale gramsciano ha il dovere etico di avvicinarsi alle classi subalterne, istillando in esse una presa di coscienza e fornendo loro strumenti reali di conoscenza della realtà per cambiarla.

Parole chiave: Gramsci. Militanza. Arresto. Solitudine. Divario.

Resumo: Com o presente artigo pretende-se, antes de tudo, recordar a figura e o pensamento de Antonio Gramsci traçando aspectos biográficos importantes para avaliar o homem na sua totalidade. Parte-se, portanto, dos episódios cruciais que contribuíram para sua formação intelectual: da infância passada na terra sarda até os agitados anos na Turim universitária e proletária; do encontro com Tania à militância comunista, da detenção até os anos de Revista Italiano UERJ 
cárcere. Exatamente no lugar de constrangimento físico máximo Gramsci elabora o ambicioso sonho comumunista, trabalhando nele incessantemente, enquanto é progressivamente abandonado por muitos companheiros de seu partido e por sua esposa (acontecimento este nunca esclarecido). Uma história de forte paixão - civil, humana, em outras palavras, política - de um lado, mas também uma história de dor, de sofrimentos e de extrema solidão do fundador do Partido Comunista Italiano que encontra na escrita a libertação para uma luta constante. O objetivo deste trabalho é também chamar a atenção para o persistente desequilíbrio presente no panorama políticocultural italiano, entre certa intelectualidade que professa a esquerda e o povo, e para a clara contradição de uma classe de casta que não se empenha para superar essa lacuna. Esta falta de vontade da esquerda é exatamente o oposto do que queria o grande pensador ao qual, mesmo que de maneira retórica, às vezes se retorna: o intelectual gramsciano tem o dever ético de se aproximar das classes mais baixas, incutindo-lhes uma consciência e fornecendo-lhes ferramentas de conhecimento efetivo da realidade para mudá-la.

Palavras-chave: Gramsci. Militância. Prisão. Solidão. Desequilíbrio.

Istruitevi, perché avremo bisogno di tutta la nostra intelligenza. Agitatevi, perché avremo bisogno di tutto il nostro entusiasmo.

Organizzatevi, perché avremo bisogno di tutta la nostra forza. (da L’Ordine Nuovo, anno I, n. 1, $1^{\circ}$ maggio 1919).

\section{Introduzione}

1.1 È molto probabile, per non dire certo, che Antonio Gramsci non avrebbe gradito le parole apposte sulla lapide a lui dedicata dai compaesani nel decennale dalla morte: 
Dieci anni dopo il suo martirio/ad antonio gramsci / dedicata/nella sua casa ove nacque/questa pietra posero/l'affetto dei concittadini/la riconoscenza degli uomini liberi.

A dirla tutta Gramsci non aveva un bel rapporto col paese di origine e non lo mantenne neppure in seguito, tranne che per gli affetti e per la cara figura materna (da cui non si sentirà mai tradito e alla quale scriverà, negli anni a seguire, lettere di intensa commozione $\left.{ }^{1}\right)$. Per di più mai avrebbe voluto essere celebrato come un eroe, tantomeno come un martire, questo si evince almeno dai numerosi scritti: una sorta di ripugnanza e per la retorica e per il vittimismo.

Credo gli sarebbe bastato essere considerato un uomo tra gli uomini, un uomo convinto delle proprie idee e ad esse fedele fino in fondo. Incarcerato non chiese mai la grazia e usò toni infuriati contro la cognata, Tatiana, che si era adoperata più volte perché la ottenesse. Mussolini, che in un discorso alla Camera, riferendosi al deputato Gramsci aveva detto "Bisogna impedire a questo cervello di funzionare", nel maggio 1933 si decide a concedergli il trasferimento al carcere di Turi solo per evitare uno scandalo internazionale, mentre in Francia già si erano costituiti comitati di solidarietà contro la sua morte. Ma le sue condizioni di salute erano ormai irreversibili e Antonio Gramsci, di lì a pochissimo, morirà.

\footnotetext{
${ }^{1}$ Celebre la lettera datata 10 maggio 1928 « Non ho mai voluto mutare le mie opinioni, per le quali sarei disposto a dare la vita e non solo a stare in prigione [...] vorrei consolarti di questo dispiacere che ti ho dato: ma non potevo fare diversamente. La vita è così, molto dura, e i figli qualche volta devono dare dei grandi dolori alle loro mamme, se vogliono conservare il loro onore e la loro dignità di uomini ».

${ }^{2}$ In carcere, nel 1936, scriverà a Delio, il primogenito parole struggenti: «Io penso sempre a voi; così immaginerò ogni mattino: ecco che i miei figli e Giulia pensano a me in questo momento. Tu sei il fratello maggiore, ma devi dirlo anche a Julik: così ogni giorno avrete i cinque minuti col babbo».
} 
È molto difficile in questo caso riuscire a distinguere l' uomo privato da quello pubblico, tanto le due dimensioni furono interrelate. D'altra parte l'adesione ideologica autentica, e radicale, richiedeva una necessaria commistione tra l'io e il noi, tra la dimensione personale e quella collettiva, esigeva una dedizione assoluta anche a costo di sacrificare tutto per la causa comune. Eppure non si può cogliere a mio avviso la profondità del pensiero gramsciano se non si ritorna ad alcuni fatti importanti che incideranno a lungo sul suo percorso, generando conflitti, angosce, solitudini diverse e tormenti interiori, nell' intellettuale e nel capo di partito, ma anche nel marito, nel cognato, nel padre che fu e in quello che avrebbe potuto e voluto essere ${ }^{2}$.

\section{L’ Infanzia sarda e le vicissitudini familiari.}

2.1 Gli viene riscontrata fin da piccolo una malformazione congenita che in casa però, per ignoranza, vergogna, o per ingenua protezione, imputano a una probabile caduta dalle braccia della balia che gli avrebbe impedito una normale crescita. In realtà Gramsci era affetto dal morbo di Pott, e già all'età di quattro anni sembrava essere spacciato ${ }^{3}$. Il lugubre presagio però non si avvera e il bambino non muore. Può essere che un evento simile abbia temprato in qualche modo il suo carattere: scampare da una morte certa doveva infondere un grande senso di lotta in un bambino sensibile e introverso, insegnandogli una primissima lezione, quella della sopravvivenza.

\footnotetext{
${ }^{3}$ Si veda LEPRE, Aurelio. Il prigioniero: vita di Antonio Gramsci. Bari: Laterza, 1998, p. 6.

${ }^{4}$ In MAURO, Walter. Invito alla lettura di Antonio Gramsci. Milano: Mursia, 1981, p. 18.

${ }^{5}$ Ivi, p. 18.
} 
2.2 Ha nove anni quando la famiglia si trova a vivere un dramma che avrà delle forte ripercussioni, su tutti e soprattutto sull'economia della casa: il padre di Antonio viene arrestato e dovrà restare in prigione per diversi anni, con l'accusa di aver manomesso la finanza pubblica. La famiglia cade improvvisamente in uno stato di forte indigenza economica, che costringe sia la madre che i giovanissimi figli a lavorare per andare avanti. La condanna e la detenzione, un «trauma umiliante» ${ }^{4}$, vengono però tenuti nascosti a Antonio, che lo viene a sapere comunque, come è naturale che sia in un piccolo paese come Ghilarza. Il fatto genera un forte senso di rancore da parte di Tonino, così lo chiamano in famiglia, soprattutto verso il padre.

Anni dopo, messo in carcere dai fascisti, si trova a dover vivere la stessa vicissitudine ribaltata: rimprovera Giulia, la moglie, per aver deciso di non dire al primogenito Delio quanto è accaduto. Antonio aveva vissuto sulla propria pelle il trauma e conosceva bene le conseguenze interiori su chi ha subito e scopre di essere stato tradito dai propri stessi familiari:

« [...] per un bambino, che incomincia a dubitare della veridicità dei suoi educatori e incomincia a pensare per conto proprio e far vita a sé $»^{5}$.

Anche qui forse si ritrovano già i primi segni di quell'amore per la verità che non lo abbandona mai più. Celebre il motto gramsciano "La verità è sempre rivoluzionaria".

\section{Torino e gli anni della militanza.}

3.1 Nel 1911 arriva a Torino e il periodo trascorso nel capoluogo piemontese resta fondamentale nella formazione del pensiero gramsciano. Gramsci è un giovane studente, è bravo e squattrinato. Nonostante la borsa di 
studio, i soldi non bastano mai e restano molte lettere con una insistente richiesta di denaro ai genitori, la cui situazione si era in parte normalizzata dopo la scarcerazione paterna senza però arrivare all'agiatezza. Nelle lettere ricorda anche il freddo patito e l'inverno passato senza soprabito, il forte stato di ansia che lo divora per il timore di perdere il Collegio, ma anche il senso di vergogna e inadeguatezza, che lo spinge spesso all'isolamento. L'ambiente dell' Ateneo torinese però è un ambiente interessante, vivo e impegnato nel dibattito culturale di quegli anni. Si presenta quindi come un milieu di forte stimolo per lui che proprio qui inizia la militanza politica e matura il proprio pensiero critico ${ }^{6}$. Sono i difficili anni del Biennio Rosso, l' Europa è scossa da sommosse, rivolte, scioperi tra le masse e i poteri costituiti. In Russia intanto, dopo la Rivoluzione d'ottobre del 1917, si instaurava il primo Stato Socialista nella storia occidentale. Anche in Italia gli operai occupano le fabbriche e Torino è la città operaia per eccellenza, il campo di battaglia delle lotte tra i lavoratori e i padroni e il posto migliore per fare una esperienza politica diretta. Il giovane studioso sardo entra nelle fabbriche, partecipa alle riunioni operaie, entra in contatto con quell'umanità di cui inizia a conoscere i bisogni radicali. Prima ancora di elaborare l'idea dell' intellettuale «organico» in un certo senso Gramsci già lo è: "tuffandosi” tra la gente abbatte ogni distanza e si mescola con loro, per loro. Sono anni di scoperte, di frequentazioni, di elaborazioni e rielaborazioni. Soprattutto sono gli anni della praxis, in cui il politico impegnato nella causa socialista si trova fianco a fianco con quegli sfruttati di cui si farà strenuo difensore per tutta la vita.La prigionia, dal 1925

\footnotetext{
${ }^{6}$ «[...] e soprattutto il passaggio da un determinismo ancora positivistico, con la mediazione del neoidealismo, ad un marxismo che mai verrà accettato dogmaticamente». Ivi, p. 25.

7 Antonio Gramsci- Tatiana Schucht, Lettere. 1926-1935, a cura di NATOLI, Aldo e DANIELE Chiara. Torino: Einaudi, 1997, p. 3.

${ }^{8}$ In LEPRE, 1998, p. 95, 97-101.
} 
al 1937, gli aprirà nuove realtà, diventerà un acuto osservatore-antropologo (i detenuti di Napoli gli sembrano «esemplari zoologici fantasmagorici») ${ }^{7}$ dei detenuti specchio delle tante Italie esistenti, e verrà a conoscenza delle leggi di potere che regolano $\mathrm{i}$ rapporti umani in quel mondo sconosciuto che genericamente definiamo sottoproletariato ${ }^{8}$.

Torino è inoltre il luogo in cui l'analisi gramsciana abbandona l'ottica isolana e si apre a una nazionale, storicamente, socialmente ed economicamente diversa dalla Sardegna. Gli anni torinesi sono scanditi da un intensissimo, febbrile attivismo, con una gran voglia di «fare, fare, fare» dirà lo stesso Gramsci, tramite l'assidua frequentazione di riunioni a carattere politico, la creazione di associazioni dalla forte impronta etica, e soprattutto l' impegno giornalistico con la fondazione di riviste. I nomi stessi dei periodici ricalcano (e proiettano) la tensione civile e morale del disegno utopico sempre più marxista, da un lato, e la necessità di rivendicare la voce delle masse inascoltate, dall'altro. Nel 1917 scrive sulla rivista «La città futura», organizza «Club di vita morale», due anni dopo è direttore di «Il Grido del Popolo». Nel 1919 nasce "L' Ordine Nuovo", soppresso poi dal regime fascista (1925), che diventerà il quotidiano comunista par excellence. Oltre ai colleghi progressisti, tra cui Togliatti, Teraccini e Tasca, Gramsci gradisce la collaborazione del liberale Gobetti, mostrando già quell'apertura di vedute, quella lungimiranza

\footnotetext{
${ }^{9} \mathrm{Da}$ «La città futura», numero unico, 11 febbraio 1917: «Odio gli indifferenti. Credo che vivere voglia dire essere partigiani. Chi vive veramente non può non essere cittadino e partigiano. L'indifferenza è abulia, è parassitismo, è vigliaccheria, non è vita. Perciò odio gli indifferenti. (...) Alcuni piagnucolano pietosamente, altri bestemmiano oscenamente, ma nessuno o pochi si domandano: se avessi fatto anch'io il mio dovere, se avessi cercato di far valere la mia volontà, sarebbe successo ciò che è successo?».
} 
che gli avrebbero procurato in seguito fortissimi disaccordi all'interno del partito Comunista italiano, soggiogato dalla intransigente linea stalinista.

3.2 Nel 1921 al Congresso di Livorno Gramsci fonda il Partito Comunista italiano e poco dopo, nel 1923, parte per Mosca come esponente del PCI nell' Esecutivo della Internazionale. Si discosta intanto dalla posizione estremista di Bordiga, che rifiuta qualsiasi connubio coi socialisti. L' Internazionale promuove la sua linea, ora prevalente nel Partito, grazie soprattutto all'appoggio dei dirigenti bolscevichi che vedono in lui "un piccolo gobbo dalla grande testa e dallo sguardo intelligente, profondo". In Russia scopre la passione amorosa che subito lo travolge, e lo spaventa:

«Quante volte mi sono domandato se legarsi ad una massa era possibile quando non si era mai voluto bene a nessuno?» Un uomo abituato all' impegno politico ma poco esperto nelle questioni di cuore si domanda se queste non avrebbero prevalso su quelle della mente.

Ricoverato per ragioni di salute in una casa di cura russa conosce Giulia, di cui si innamora a priva vista. Il dolce ricordo dell incontro a Serebriani Bor lo accompagnerà negli anni più difficili dandogli, a tratti, un conforto. Il tragico equivoco mai chiarito tra i due, durante la detenzione, per i lunghi silenzi di Giulia e le sue lettere tenute in borsa e mai speditegli, alimentano in lui stati ossessivi e paranoici, insieme a un profondo risentimento e senso di abbandono ${ }^{10}$. Le scrive:

\footnotetext{
10 Interessante la prospettiva dell'autrice Laura Tancredi, che avanza una nuova lettura di Giulia Schucht, una donna “(...) rimossa, condannata dalla Storia ad estinguersi ". Si veda: TANCREDI, Laura. La vita privata di Giulia Schucht. Macerata: Ev casa editrice, 2012.

10 La lettera da lui inviata a Togliatti nel 1926 sancisce la definitiva rottura tra i due. Da quel momento Gramsci viene messo ai margini dal suo stesso partito.

${ }^{11}$ Per un approfondimento sulla questione meridionale si consiglia LEPRE, op. cit., 1998, p. 97 e ss.
} 
«Mi pare, e lo dico anche se devo farti provare un forte dispiacere, che tu hai contribuito ad aggravare il mio isolamento, facendomelo sentire più amaramente».

L' anno successivo (1924) viene eletto deputato e, contestualmente, fonda il quotidiano politico l' «Unità», che diventa organo del PCI. Al Congresso di Lione del 1926 la maggioranza è ormai dalla sua parte e mantiene la nomina di Segretario generale del Partito. Nelle «Tesi di Lione», pronunciate durante il convegno, sono già presenti i capisaldi del suo programma che, al momento, coincidono con quelli del Partito ${ }^{11}$. La circostanza per elaborare il grande sogno della praxis si presenta a Gramsci di lì a poco. In carcere, rinchiuso tra le quattro mura della cella, egli vi si dedica fino alla fine: quanto più le sue condizioni fisiche e psichiche peggiorano, tanto più cresce in lui la determinazione assoluta e il senso estremo di abnegazione.

\section{L'arresto e l'elaborazione del pensiero gramsciano.}

4.1 Intanto stava scrivendo un saggio sulla questione meridionale ${ }^{12}$, ed era ricercato dalla polizia fascista per la presunta attività politica sovversiva. L' 8 novembre del 1926, viene portato in carcere, nonostante l' immunità parlamentare cui pure avrebbe diritto. La moglie intanto, incinta del secondogenito, era tornata a Mosca, ormai stalinizzata. Non si rivedranno mai più e Antonio Gramsci non conoscerà mai il secondo figlio, Giuliano.

Condotto a Regina Coeli, poi Ustica e di qui a San Vittore, nel maggio del 1928 viene processato e condannato dal Tribunale speciale per la difesa dello Stato alla pena di venti anni, quattro mesi e cinque giorni di reclusione. A 
partire dal luglio 1928 viene trasferito a Turi, in provincia di Bari, e qualche mese dopo gli viene accordato il permesso di scrivere e lavorare in carcere. Informa la cognata-compagna, che è il suo tramite col partito e con il mondo, del suo progetto:

«Vorrei, secondo un piano prestabilito, occuparmi intensamente e sistematicamente di qualche oggetto che mi assorbisse e centralizzasse la mia vita interiore».

I numerosi scritti della reclusione, non destinati alla pubblicazione, sono dati alle stampe dall'editore Einaudi, insieme alle Lettere ai familiari, soltanto nel secondo dopoguerra, con sollecitudine (e controllo?) del dirigente comunista Felice Platone. I Quaderni del carcere, in sei volumi ${ }^{13}$, vengono sistemati per temi affini, così intitolati: Il materialismo storico e la filosofia di Benedetto Croce (1948), Gli intellettuali e l'organizzazione della cultura (1949), Il Risorgimento (1949), Note sul Machiavelli, sulla politica e sullo Stato moderno (1949), Letteratura e vita nazionale (1950), Passato e presente (1951). Nel 1975 esce una nuova edizione a cura di Valentino Gerratana, strutturata secondo un ordine cronologico $^{14}$.

4.2 Nell' impossibilità di descrivere in dettaglio la Weltanschauung pratica gramsciana presente nei Quaderni, scelgo di soffermarmi su alcuni punti

\footnotetext{
${ }^{13}$ Poi c'è l'annosa polemica del Quaderno fantasma per cui si rimanda ai seguenti articoli: PONS, Silvio, Gramsci tradito? Nuovi indizi contro Togliatti (Lettera inedita da Mosca), Corriere della sera, 17 luglio 2003; CANFORA, Luciano, Attenti ai dossier del compagno Stalin. E occhio alle date, Corriere della sera, 19 luglio 2003; PONS, Silvio, Togliatti, Gramsci e l'ombra del tiranno, Corriere della sera, $27 \quad$ luglio 2003; CANFORA, Luciano, Inedito su Gramsci, i punti aperti, Corriere della sera, 30 luglio 2003.

14 Sono stati raccolti in volume anche tutti gli articoli scritti da Gramsci nell' «Avantil», in «Il Grido del popolo» e in «L' Ordine Nuovo».
} 
che ritengo importanti per fornire un quadro, se non esaustivo, almeno chiaro.

Nello Stato Nuovo si sarebbe anzitutto dovuto creare un blocco tra le forze sociali, formato dall'unione della classe operaia del Nord con le masse contadine del Sud. Al Partito sarebbe spettato il compito di coordinare e militare il gruppo, per condurlo, progressivamente, verso la rivoluzione e la meta finale: la dittatura del proletariato. Secondo la prospettiva marxistagramsciana solo attraverso la "dittatura del proletariato", regolamentata al suo interno, le masse si sarebbero liberate finalmente dalle secolari ingiustizie e oppressioni subite. La nuova classe al potere avrebbe cosi sostituito i vecchi sistemi economici e culturali borghesi imponendo propri modelli, riflesso dei propri bisogni materiali. La preparazione di una rivoluzione radicale pone questioni serie sulla organizzazione della cultura nuova. Come si possono preparare i dominati che dovranno un giorno dominare? Il compito spetta in primis all intellettuale, dice Gramsci, «organico», impiegato dal Partito a infondere la presa di coscienza nelle masse, perché diventino cellule di un unico organismo. Il nuovo intellettuale deve sostituire quello tradizionale, incarnato dagli intellettuali post-crociani suoi coetanei. Il suo compito è funzionale al risveglio dal torpore dell' ignoranza e della rassegnazione, alla volta di un riscatto corale e "di classe". L'intellettuale, maieuticamente, deve tirar fuori quello che è già presente in potenza in ogni singolo proletario, perché è necessario che sia organizzato e sicuro di sé una volta preso il potere e ciò è possibile, secondo il non dogmatico Gramsci, perché « (...) non si può separare l'homo faber dall'homo sapiens». Di qui l' insistenza sul valore dell'educazione unita all'osservazione di una mancanza in Italia, a differenza degli altri paesi europei, di una letteratura che lui chiama "nazional-popolare". Osserva: 
(...) in Italia, il termine naz̧ionale ha un significato molto ristretto ideologicamente e in ogni caso non coincide con popolare, perché in Italia gli intellettuali sono lontani dal popolo, cioè dalla nazione e sono invece legati a una tradizione di casta, che non è mai stata rotta da un forte movimento popolare o nazionale dal basso: la tradizione è libresca e astratta e l'intellettuale tipico moderno si sente più legato ad Annibal Caro o a Ippolito Pindemonte che a un contadino pugliese o siciliano ${ }^{15}$.

A parte il melodramma, in Italia è mancata una letteratura popolare, e c' è stata invece una spaccatura profonda tra l'alto e il basso, tra la società letterata e quella delle masse, tra il pubblico e gli scrittori. Nei Quaderni si legge:

«(..) Le Accademie il simbolo, spesso a ragione deriso, del distacco esistente tra l'alta cultura e la vita, tra gli intellettuali e il popolo ${ }^{16} »$.

Il compito del nuovo letterato gramsciano è proprio quello di colmare la frattura ed elevare le masse liberando l'Italia dall' egemonia di tanta letteratura straniera. In questo hanno fallito finora gli intellettuali laici, come i cattolici che in particolare hanno lasciato il popolo in una arretratezza superstiziosa post-tridentina ${ }^{17}$. Tra gli scrittori di punta della storia letteraria italiana tramandata Gramsci individua un paio di modelli antitetici, da un lato Manzoni, dall'altro Machiavelli. Il primo esprimerebbe il carattere aristocratico

15 GRAMSCI, Antonio, Quaderni dal carcere a cura di V. GERRATANA. Torino: Einaudi, 1975, p. 127.

16 GRAMSCI, Antonio, Quaderni del carcere, Gli intellettuali e l'organizzazione della cultura. Torino: Einaudi, 1966 p. 103.

${ }^{16}$ In GRAMSCI, 1975, p. 131. 
della letteratura italiana e l'atteggiamento paternalista cattolico verso il popolo. Il secondo invece viene valutato come il primo rappresentante di un mondo non più teocratico come quello medievale, ma empirico e terreno.

L'elaborazione di un nuovo tipo di intellettuale pone inevitabilmente Gramsci in un rapporto polemico e dialettico con la classe intellettuale italiana dei primi quindici anni del ventesimo secolo. ${ }^{18}$ Egli considera positivamente il primo Croce, di cui ammira soprattutto lo spirito, l'ansia rinnovatrice e laica che lo accompagna. Scrive alla cognata in una lettera del 1931:

partecipavamo in tutto o in parte al movimento di riforma morale e intellettuale promosso in Italia da Benedetto Croce, il cui primo punto era questo, che l'uomo moderno può e deve vivere senza religione rivelata o positiva o mitologica o come altro si vuol dire. Questo punto anche oggi mi pare il maggior contributo alla cultura mondiale che abbiano dato gli intellettuali moderni italiani.

Se ne allontana invece rispetto alle concezioni dell'autonomia dell'arte e dell'intellettuale super partes, rivendicata dal critico abruzzese. Gramsci ha in mente esattamente il contrario, per lui l'arte deve essere vincolata a un credo, deve essere utile e funzionale a uno scopo comune e l'intellettuale non può stare al di fuori delle cose, ma deve farne assolutamente parte. Egli si pone piuttosto in continuità con la linea tracciata da De Sanctis, paradigma del fervore etico e civile risorgimentale. Quella di De Sanctis è una critica

\footnotetext{
${ }^{18} \mathrm{Nei}$ Quaderni si propone di scrivere un saggio chiamato I nipotini di padre Bresciani, in riferimento agli epigoni di padre Bresciani, per cui riserva epiteti poco carini, fondatore di «La Civiltà Cattolica» e autore di romanzi di impronta conservatrice già stroncati da De Sanctis.
} 
militante, non solo estetica, e per questo viene giudicata favorevolmente, poiché rappresenta la lotta per costruire una nuova cultura.

4.3 Gramsci muore il 27 aprile del 1937, nemmeno una settimana dopo dall'uscita del carcere. Le sue ceneri vengono portate al Cimitero degli Inglesi a Roma. Al funerale sono presenti solo Tatiana Schucht e Carlo, il fratello, nessun altro. Palmiro Togliatti, ex compagno di studi e di partito, è assente. Per una strana coincidenza il suo funerale avrebbe dovuto essere celebrato il primo maggio. È documentato ormai che $\mathrm{i}$ fascisti impedirono che le esequie ricadessero nello stesso giorno della festa dei lavoratori, abolita dal regime mussoliniano, per timore che si scatenasse un corteo dissenziente ${ }^{19}$.

\section{Considerazioni finali.}

5.1 Nonostante la ripresa e il susseguente boom degli studi gramsciani a partire dagli anni Sessanta del secolo scorso, credo che l' insegnamento di Antonio Gramsci non sia stato assimilato da buona parte della sinistra italiana. $\mathrm{Si}$ è trattato piuttosto di un recupero formale, non sostanziale, che ha generato blande ipocrisie pseudo-marxiste con conseguenze nefaste, sia sul versante politico che su quello intellettuale. $\mathrm{Da}$ un lato lo scollamento tra una società politica che dice e poi non fa (o non fa quello che dovrebbe fare), e quella civile, dall'altro persevera, diabolica, la spaccatura tra il sistema culturale e il popolo. Mi pare che molti degli intellettuali che si autoproclamano "di sinistra" siano ancora elitari, vivendo e scrivendo nelle loro torri d'avorio.

[...]Gli intellettuali non escono dal popolo, anche se accidentalmente qualcuno di essi è di origine popolana, non si sentono legati ad esso (a parte la retorica), non ne conoscono e non ne sentono i bisogni, le aspirazioni, i

\footnotetext{
19 Si legga in proposito: CARLUCCI, Davide, Gramsci ceneri vietate, La Repubblica, 25 Aprile 2007.
} 
sentimenti diffusi; ma, nei confronti del popolo, sono qualcosa di staccato, di campato in aria, una casta, cioè, e non un'articolazione, con funzioni organiche, del popolo stesso ${ }^{20}$.

Gramsci è stato un grande esempio, non ha desistito dalla lotta e ha continuato a combattere, giorno e notte, perché il sogno comune è un sogno grande e ambizioso e bisogna lavorarci incessantemente.

Sono grata ai fascisti per un piccolo, grande errore di calcolo. Malaticcio, devono aver pensato che sarebbe bastato togliergli la libertà fisica per eliminarlo. Sbagliavano. Avrebbero dovuto privarlo della penna. Tanto gli è bastato per segnare una svolta nella storia del pensiero.

\section{Bibliografia}

CANFORA, Luciano. Attenti ai dossier del compagno Stalin. E occhio alle date, Corriere della sera. 19 Luglio 2003.

Inedito su Gramsci, i punti aperti, Corriere della sera. 30 luglio 2003.

CARLUCCI, Davide. Gramsci ceneri vietate, La Repubblica. 25 luglio 2007.

${ }^{20}$ Letteratura popolare, in GRAMSCI, 1950, pp. 135-140. 
GRAMSCI, Antonio. Letteratura e vita nazionale. Torino: Einaudi, 1950 (nuova ed. Roma: Editori Riuniti, 1971, da cui si cita).

Quaderni del Carcere, Gli intellettuali e l'organizzazione della cultura. Torino: Einaudi, 1966.

. Quaderni del carcere, a cura di V. GERRATANA. Torino: Einaudi, 1975.

. Tatiana Schucht Lettere 1926-1935. Torino: Einaudi, 1997.

LEPRE, Aurelio. Il prigioniero, Vita di Antonio Gramsci. Bari: Laterza, 1998.

MAURO, Walter. Invito alla lettura di Antonio Gramsci. Milano: Mursia, 1981.

ORVIETO, Paolo. L'area marxista: teorici e critici. In BIAGINI, E. , BRETTONI, A.; ORVIETO, P. Teorie critiche del Novecento. Roma: Carocci, 2001.

PONS, Silvio. Gramsci tradito? Nuovi indizi contro Togliatti (Lettera inedita da Mosca), Corriere della sera. 17 luglio 2003.

Togliatti, Gramsci e l'ombra del tiranno, Corriere della sera. 27 luglio 2003. 
TANCREDI, Laura. La vita privata di Giulia Schucht. Macerata: Ev casa editrice, 2012.

TELLINI, Gino. Metodi e protagonisti della critica letteraria. Milano: Mondadori, 2010. 